For Submission to Materials Science \& Engineering A, Nov 2015, Revised Feb 2016

\title{
Effect of microstructure on strain localization in a 7050 Aluminum alloy: comparison of experiments and modeling for various textures
}

\author{
Alberto W. Mello ${ }^{1}$, Andrea Nicolas ${ }^{1}$, Ricardo A. Lebensohn², Michael D. Sangid ${ }^{1, *}$ \\ ${ }^{1}$ School of Aeronautics and Astronautics, Purdue University, 701 W. Stadium Ave, West \\ Lafayette, IN 47907-2045, USA \\ ${ }^{2}$ Materials Science and Technology Division, Los Alamos National Laboratory, Los \\ Alamos, NM 87545, USA \\ * Corresponding Author, e-mail address: msangid@purdue.edu
}

\begin{abstract}
Microstructure attributes are responsible for heterogeneous deformation and strain localization. In this study, the relation between residual strain fields and microstructure is examined and assessed by means of experiments and crystal plasticity modeling. The microstructure of rolled aluminum alloys (AA) in the 7050T7451 condition was experimentally obtained with electron backscatter diffraction (EBSD) analysis along the rolling direction (L-T orientation), across the rolling direction ( $\mathrm{T}-\mathrm{L}$ orientation), and transverse to the rolling direction (T-S orientation). Each of these sections was also patterned using a novel microstamping procedure, to allow for strain mapping by digital image correlation (DIC). The measured microstructures were in turn used as input of an elasto-viscoplastic crystal plasticity formulation based on fast Fourier transforms (EVP-FFT). Comparisons between the strain maps obtained experimentally by the concurrent DIC-EBSD method and the EVP-FFT simulations were made for the three sections, corresponding to the initial textures. The comparisons showed that the predicted levels of strain concentration were reasonable for all three specimens from a statistical perspective, which is important to properly describe and predict the strains within an ensemble of components; however the spatial match with the actual strain fields needs improvement.
\end{abstract}

Keywords: microstructure; digital image correlation; electron backscatter diffraction; strain localization; heterogeneous deformation; elasto-viscoplastic crystal plasticity.

\section{Introduction}

Aluminum alloys play an important role in the modern transportation industry, due to their combination of weight, strength, ease of manufacture, and environmental resistance. In worldwide aviation, aluminum alloys are present in more than two thirds 
of the plane's dry weight, still being the preferred material for an aircraft's primary structures. It means that the majority of the load carrying components and fatigue critical locations are made of this material, which are frequently stressed in multiple directions and under complex loading conditions. Component failure is a result of deformation accumulating in small regions within a part. In fact, strain localization is a precursor to material failure. Understanding the strain localization and the role of microstructure, e.g. grain's orientation and boundaries, on the strain energy accumulation is a key factor for improving the application of such materials under aggressive loading and environments. In this paper, the investigation of strain localization is enabled through combined experimental analysis and material's modeling, the outcomes of which are explored and compared.

In polycrystalline materials, the microstructure attributes are responsible for heterogeneous deformation. The presence of grains and grain boundaries tends to localize deformation. Digital image correlation (DIC) has become a valuable technique to study local strain in materials and components through non-contact/non-destructive analysis. Additionally, electron backscatter diffraction (EBSD) is the predominant technique to identify spatial maps of local grain orientations. In recent years, microstructural information has been coupled with local strain maps by means of concurrent DIC-EBSD. For example, Tschopp et al. performed in-situ strain mapping in a scanning electron microscope (SEM) of a Ni-base superalloy, Rene 88DT [1]. Clair et al. used kernel average misorientation to investigate local strain near triple points [2]. Abuzaid et al. used DIC-EBSD to investigate polycrystalline deformation [3]. In this case, an ex-situ technique was used at $31 x$ magnification to investigate a large area of interest $(1 \mathrm{~mm}$ by $0.8 \mathrm{~mm})$. The results were analyzed using Taylor model, to identify the individual slip systems that accommodated plastic deformation. Carter et al. performed DIC in an SEM at elevated temperatures to study grain boundary sliding mechanisms [4]. Kammers and Daly analyzed deformation in ultra-fine grain material with significant contributions to state-of-the-art speckle patterns and biasing and calibrations necessary for the quantification of strain fields obtained from DIC within an SEM [5]. Da Fonseca et al. used a novel speckle pattern technique achieving slip level resolution in their strain maps [6]. Esquivel and Sangid have used DIC-EBSD technique within a SEM to resolve the strain accommodated within individual slip bands [7].

Elasto Viscoplastic crystal Plasticity (EVP) links the applied macroscopic load and micro-mechanical response, accounting for slip activation. Experimental strain maps have been recently compared with crystal plasticity simulations. Using an oligocrystal aluminum sample, Zhao et al. suggested that grain topology and microtexture have significant influence on the origin of strain heterogeneity [8]. Turner et al. carried out a detailed comparison between DIC (enabled by individual strain gauges produced by focus ion beam providing fiducial marks on the specimen surface) and model predictions, and pointed out the wide variation in mechanical behavior produced by the subsurface microstructure [9]. Further, Tasan et al. used similar comparisons between modeling and experiments on dual phase steels to identify hot spots of 
damage at locations of larger ferritic grains and lower local martensitic fractions [10]. Lim et al. concluded that EVP model predictions agree reasonably well at various applied strains in tantalum oligocrystals [11]. In the present work, various orientations of highly textured, rolled Al plate are characterized and compared with EVP simulations performed with direct input from microstructural images using an FFT-based model framework. The work demonstrates the EVP-FFT method accurately reproduces the statistical nature of heterogeneous deformation of the various configurations, but cannot capture the exact strain localization at individual microstructure features.

\section{Material and Method}

\subsection{Material}

A plate of 7050 aluminum alloy (AA) received in the T7451 condition with nominal composition [12] was used in this study. Three specimens were machined from the plate, with a length of $48 \mathrm{~mm}$ in the following directions: parallel to the rolling direction of the plate $(\mathrm{L}-\mathrm{T})$; perpendicular to the rolling direction, with the width aligned with the long direction (T-L); and perpendicular to the rolling direction, with the width aligned with the short (thickness) direction (T-S), as shown in Fig. 1. All the specimens were $1.6 \mathrm{~mm}$ thick and machined $6.4 \mathrm{~mm}$ away from the plate surfaces to avoid the excessive effect of the rolling process. The specimen geometry was adapted from the ASTM E8 [13] standard, to better serve the purpose of the DIC characterization. The geometry of specimens chosen for the present study was based on the size of the surface to be analyzed and compatible dimensions with the SEM chamber.

\subsection{Experimental Procedures}

All tension experiments were conducted at room temperature $\left(23^{\circ} \mathrm{C}\right)$, following the basic procedures described in [13]. For the DIC experiments, the specimens were polished on 1200 grit sand paper for 2 to 3 minutes or until all machining marks were completely removed. One side of each specimen was then polished for 40 minutes using a NAPAD with $0.05 \mu \mathrm{m}$ blue colloidal silica, or until a mirror-like surface was obtained. After final polishing, the area of interest was properly marked (using fiducial indents) in the center of the specimen [14], as depicted in Fig. 2. For placing the markings on the specimen, the automated LECO Microhardness Tester LM247AT was used. The two center indents were obtained with $1 \mathrm{~N}$ of indentation force, and the four smaller indents in each side defining the areas of interest were obtained with $0.5 \mathrm{~N}$. Each area of interest was defined by fiducial markings in a rectangle of $800 \mu \mathrm{m}$ by 600 $\mu m$. 



Figure 1. Specimen geometry and orientations from AA 7050-T7451 plate (dimensions in $\mathrm{mm}$ ). Each specimen had a length of $48 \mathrm{~mm}$ and a thickness of $1.6 \mathrm{~mm}$.

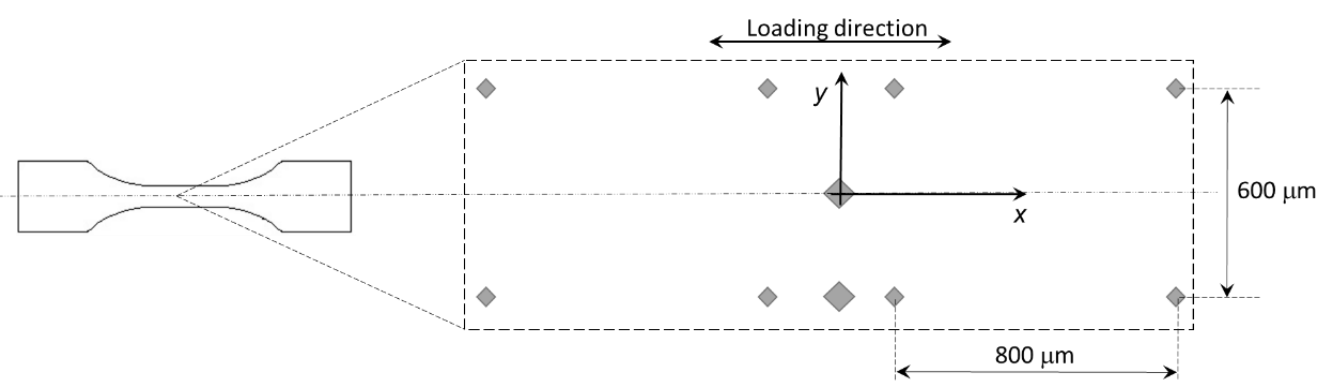

Figure 2. Delimited area for EBSD and DIC analysis

An FEI Philips XL-40 SEM was used for EBSD characterization. The average grain size was $80 \mu \mathrm{m}$, and typically ranging from 30 to $500 \mu \mathrm{m}$, depending on the specimen orientation with respect to the alloy rolling direction. For DIC patterning, the specimens were stamped using a novel micro stamp, manufactured by 1900 Engineering LLC, designed for the DIC reference patterning [15]. Details of the flexible micro textured stamps are outlined in [16]. A master is first created by lithography or another manufacturing method; here an e-beam lithography (EBL) process is used to create a $10 \mu \mathrm{m}$ base-element size for generating the stamp over a $12.7 \mathrm{~mm} \times 12.7 \mathrm{~mm}$ area, with a speckle population of $22 \%$. Then a castable material is selected, which should conform to sub-micron features in the master, yet should be sufficiently flexible to release from the master during the demolding step without damaging the master. The castable material is polymerized, and so the polymerization chemistry must be selected to avoid bonding to the master itself. The material is vacuum cast to the master, expelling all entrapped gas and allowing the material to flow into the sub-micron features in the master. The castable material polymerizes without shrinking, which would alter the precise microtextures to be replicated. Once the castable material is 
polymerized without bonding to the master, it is peeled from the master and can be used as a flexible micro textured stamp many times.

The following protocol briefly described by Cannon et al. [17], and repeated here for completeness: (1) Sonicate the specimen in acetone, then isopropyl alcohol for 3 minutes, each and dry; (2) With a fine liner, apply MicroChem (MCC) Primer 80/20 on the clean, polished specimen. The MCC Primer $80 / 20$ is based on a combination of $20 \%$ HMDS and $80 \%$ PM Acetate. HMDS is the best-known chemical pretreatment for increasing photoresist adhesion to difficult surfaces. PM Acetate acts as an effective pre-wetting agent; (3) Let stand for $15 \mathrm{~s}$ and gently apply compressed air from the top; (4) Bake for $3 \mathrm{~min}$ at $115^{\circ} \mathrm{C}$ on a hot plate, then remove the specimen and allow to cool to room temperature. (5) Let the hot plate cool to $60^{\circ} \mathrm{C}$, (6) Place two specimens side by side (to allow level stamping - the procedure can be applied to one specimen); (7) Apply Microdeposit Shipley 1805 photo resist on one specimen with a small pipette; (8) Wait $20 \mathrm{~s}$; (9) Apply the Shipley on the same specimen; (10) Align the stamp by touching first the dummy specimen and then let the stamp lay down over the specimen to be stamped. (11) Adjust the hot plate for $115^{\circ} C$; (12) Place a piece of cook paper over the stamp, to allow a non-stick surface for the weight; (13) Apply weight ( 4 psi); (14) Bake for 3 minutes; (15) Remove the weight and the specimen from the hot plate; (16) Carefully peel the stamp off the specimen; (17) Check the gauge section on the microscope for the patterns. The resulting speckle pattern for the $10 \mu \mathrm{m}$ pattern is shown in Fig. 3; this procedure was adopted to stamp all the AA 7050-T7451 specimens. If the pattern is not good enough over the area of interest, the stamping process has to be repeated beginning by thoroughly cleaning the specimen and stamp by repeating Step 1 . The large dark spots regularly spaced, shown in Fig. 3, are the fiducial markings, as depicted in Fig. 2. The smaller dark spots are precipitates that can be seen through the Shipley photo resist stamp.

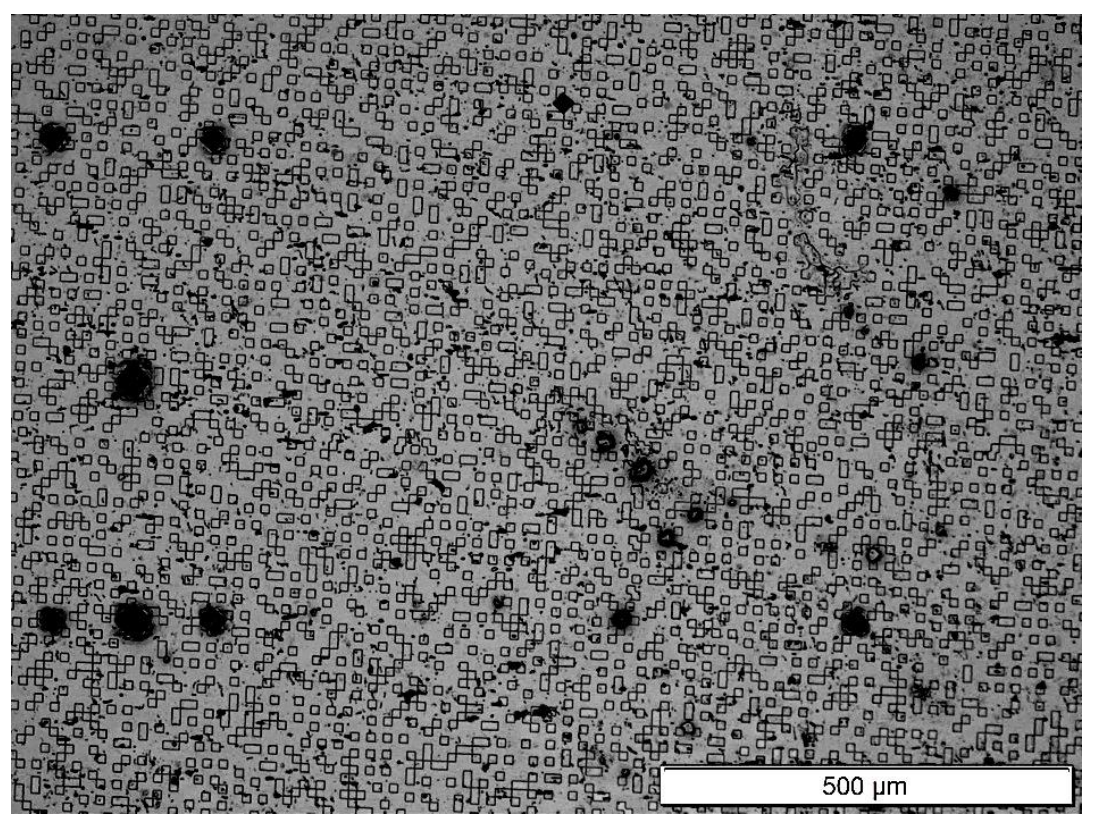

Figure 3. Area of interest viewed at 20x in optical microscope, $10 \mu \mathrm{m}$ base pattern. 
Specimens with three different orientations from the rolling direction were loaded up to rupture to determine mechanical properties and to obtain the stress-strain curves for the crystal plasticity model parameter calibration. All tension tests were performed in a $6.7 \mathrm{kN}$ electromechanical Mark-10 ESM-1500 Force Test Stand. The force indicator has a $\pm 0.1 \%$ of full scale accuracy with a resolution of $5 N$. The cross head displacement has a travel resolution of $0.02 \mathrm{~mm}$, and the tests were conducted at $2 \mathrm{~mm} / \mathrm{min}$. A dedicated Epsilon extensometer Model 3542 was used to measure strain. Six specimens were tested under tension, two for each direction (L-T, T-L, T-S as indicated in Fig. 1), with the stress-strain results shown in Fig. 4. As can be seen, the mechanical properties obtained for AA 7050-T7451 specimens are very similar in the three tested directions. The average yield stress for the L-T specimen is slightly greater than the ones for the T-L and T-S specimens. The other difference seen in these experiments that may be considered beyond the typical scatter is the final elongation of the L-T specimens that is smaller than the other two tested directions. This can be explained by the fact that the material was previously plastically deformed in the longitudinal direction during the rolling process to comply with the T7451 condition, overcoming some of its total possible elongation. On the other hand, this process increases the yield stress and it is well known to also increase the fracture toughness for this direction. The mechanical properties for the three textured samples are summarized in the Appendix, Table 2.



Figure 4. Stress-strain curves - AA 7050-T7451, $3 \mathrm{~mm}$ by $1.6 \mathrm{~mm}$ cross section. 


\subsection{Theoretical Background}

An elasto-viscoplastic EVP-FFT formulation was used to model the behavior of FCC polycrystals under uniaxial loading [18] based on the original formulation by Moulinec and Suquet [19]. The viscoplastic strain rate $\dot{\varepsilon}^{p l}$ is related with the stress $\sigma(x)$ at a single crystal material point $x$ as:

$$
\dot{\varepsilon}^{p l}(x, \sigma)=\dot{\gamma_{0}} \sum_{\alpha=1}^{N} M^{\alpha}(x)\left(\frac{\left|M^{\alpha}(x): \sigma(x)\right|}{\tau_{0}^{\alpha}(x)}\right)^{n} \operatorname{sgn}\left(M^{\alpha}(x): \sigma(x)\right)
$$

where $\dot{\gamma}_{0}$ is the reference shear rate, $\tau_{0}^{\alpha}(x)$ is the Critical Resolved Shear Stress (CRSS), which gets incrementally updated due to strain-hardening, $\mathrm{n}$ is the stress exponent, $M^{\alpha}$ is the Schmid Tensor, and $N$ is the total number of active slip systems with each slip system denoted with an index of $\alpha$.

Considering elasto-viscoplastic behavior and using an Euler implicit time discretization scheme and Hooke's law, the stress in material point $x$ and time $t+\Delta t$ (at which, unless otherwise noted, all fields are evaluated) becomes:

$$
\sigma(x)=C(x): \varepsilon^{e l}(x)
$$

where

$$
\varepsilon^{e l}(x)=\varepsilon(x)-\varepsilon^{p l, t}(x)-\dot{\varepsilon}^{p l}(x, \sigma) \Delta t
$$

with the supraindex $t$ indicating field values evaluated at time $t$. Here $\sigma(x)$ is the Cauchy stress tensor, $C(x)$ is the elastic stiffness tensor; $\varepsilon(x), \varepsilon^{e l}(x), \varepsilon^{p l}(x)$ are the total, elastic and plastic strain tensors, and $\dot{\varepsilon}^{p l}(x)$ is the plastic strain-rate tensor given by Eq. (1).

The inverse relation of Eq. (3) thus is:

$$
\varepsilon(x, \sigma)=C^{-1}(x): \sigma(x)+\varepsilon^{p l, t}(x)+\dot{\varepsilon}^{p l}(x, \sigma) \Delta t
$$

Adding and subtracting from the stress tensor an appropriate expression involving the stiffness of the reference linear medium $C_{i j k l}^{o}$ gives:

$$
\sigma_{i j}(x)=\sigma_{i j}(x)+C_{i j k l}^{o} u_{k, l}(x)-C_{i j k l}^{o} u_{k, l}(x)
$$

where $u_{k, l}(x)$ is the displacement gradient tensor. Equation (5) can be rearranged to give the stress tensor as

$$
\sigma_{i j}(x)=C_{i j k l}^{o} u_{k, l}(x)+\varphi_{i j}(x)
$$

with $\varepsilon_{k, l}(x)=\left(u_{k l}(x)+u_{k l}(x)\right) / 2$, and where the polarization field $\varphi_{i j}(x)$ is given by:

$$
\varphi_{i j}(x)=\sigma_{i j}(x)-C_{i j k l}^{o} \varepsilon_{k l}(x)
$$

If we consider equilibrium, from Eq. (6) we obtain 


$$
\sigma_{i j, j}(x)=C_{i j k l}^{o} u_{k, l j}(x)+\varphi_{i j, j}(x)=0
$$

Solving this partial differential equation (PDE) in a periodic unit cell under applied strain $E=\langle\varepsilon(x)\rangle$, the Green's function method's auxiliary PDE reads:

$$
C_{i j k l}^{o} G_{k m, l j}\left(x-x^{\prime}\right)+\delta_{i m} \delta\left(x-x^{\prime}\right)=0
$$

where $G_{k m}\left(x-x^{\prime}\right)$ is the Green's function associated with the displacement field $u_{k}(x)$. The displacement gradient can then be obtained as a convolution in real space:

$$
u_{k, l}(x)=\int_{R^{3}} G_{k i, j l}\left(x-x^{\prime}\right) \varphi_{i j, j}\left(x^{\prime}\right) d x^{\prime}
$$

Integrating by parts and assuming that the boundary terms vanish:

$$
\tilde{u}_{i, j}(x)=\int_{R^{3}} G_{i k, j l}\left(x-x^{\prime}\right) \varphi_{k l}\left(x^{\prime}\right) d x^{\prime}
$$

Equation (11) can be solved in the Fourier space as a product instead of an integral, thus shortening the computational time required for the simulation, i.e.

$$
\hat{u}_{i, j}(x)=\hat{\Gamma}_{\mathrm{ijkl}}(\xi) \hat{\varphi}_{k l}(\xi)
$$

where the Green operator is defined as $\Gamma_{\mathrm{ijkl}}=G_{i k, j l}$. The strain field becomes:

$$
\varepsilon_{i j}(x)=E_{i j}+F T^{-1}\left(\operatorname{sym}\left(\hat{\Gamma}_{\mathrm{ijkl}}(\xi)\right) \hat{\varphi}_{k l}(\xi)\right)
$$

The symbol " $\wedge$ " indicates a Fourier transform and $\xi$ is a frequency of Fourier space. The Green operator in Fourier space, which is only a function of the reference stiffness tensor $C_{i j k l}^{o}$ and the frequency $\xi$, is given by:

$$
\hat{\Gamma}_{\mathrm{ijkl}}(\xi)=-\xi_{j} \xi_{l} \hat{G}_{i k}(\xi)
$$

where

$$
\widehat{G}_{i j}(\xi)=\left[C_{i j k l}^{o} \xi_{j} \xi_{l}\right]^{-1}
$$

Next, the strain field obtained from Eq. (13) is replaced in Eq. (4) and a nonlinear system of equations is solved at every point to obtain a new guess for the stress field. With these new values of the micromechanical fields, the polarization is then iteratively updated (see Eq. (7)), and an augmented Lagrangian scheme is used to obtain, at the end of this iterative process, a compatible strain and an equilibrated stress fields [20].

The EVP-FFT formulation allows the implementation of different microscopic hardening laws without the need of changing the algorithm. The constitutive relationship used in this particular modeling was the Generalized Voce Hardening Law, which states that: 


$$
\tau(\Gamma)=\tau_{o}+\left(\tau_{1}+\theta_{1} \Gamma\right)\left[1-e^{-\frac{\Gamma \theta_{0}}{\tau_{1}}}\right]
$$

where $\Gamma(x)=\Gamma^{\mathrm{t}}(x)+\sum_{\alpha=1}^{N}\left|\dot{\gamma}^{\alpha}(x)\right| \Delta t$ is the accumulated plastic shear at material point $x, \tau_{0}$ and $\theta_{0}$ are the initial yield stress and hardening rate, respectively, and $\tau_{1}$ and $\theta_{1}$ are the parameters that describe the asymptotic behavior of the material. This hardening law is isotropic; i.e., each slip system hardens at the same rate.

An initial guess of the parameters was obtained from the macroscopic stressstrain curves, as there is a direct relationship between the microscopic and macroscopic curves through the average Taylor factor of the polycrystal, assuming this factor as constant during deformation [21]. The average Taylor factor used for the initial guess was determined from EBSD analysis (as discussed in Section 3), which corresponds to the T-L grain orientations. The final values were then obtained through manual fitting so that one set of parameters would account for all stress-strain curves. The microstructure was reconstructed from the EBSD scans by using the software Dream3D [22].

\section{Experimental Strain Field Mapping}

The method used for mapping the strain field was ex-situ digital image correlation (DIC), which consisted of characterizing the grain orientation within the areas of interest through EBSD and stamping each specimen according to the protocol described in Section 2.2. The L-T, T-L, and T-S specimens were loaded to a prescribed strain level of approximately $3 \%$, then unloaded and removed from the tensile load frame for the analysis. The strain mapping was based on the residual strain field after plastic deformation. The DIC technique has proven to be an appropriate method for full-field strain measurements [3,14,23]. Fig. 5 shows the inverse pole figures for the specimens L-T, T-L, and T-S, respectively. For all the images, the horizontal direction represents the load direction in the specimen coordinate system. As expected, the L-T, T-L, and T$S$ specimens have a predominance of elongated grains in the horizontal, vertical, and out of the plane directions, respectively. For the T-S specimen, this conclusion comes from relatively smaller grains seen in the IP figure compared with the other two directions, resulted from the grain elongation perpendicular to the plane of the image. The average grain sizes for each scan are 88, 79, and $59 \mu \mathrm{m}$ for the specimens L-T, T$\mathrm{L}$, and T-S, respectively. The Taylor factor analysis for each of the scanned materials were $\sim 2.44$, thus displaying texture due to rolling. 

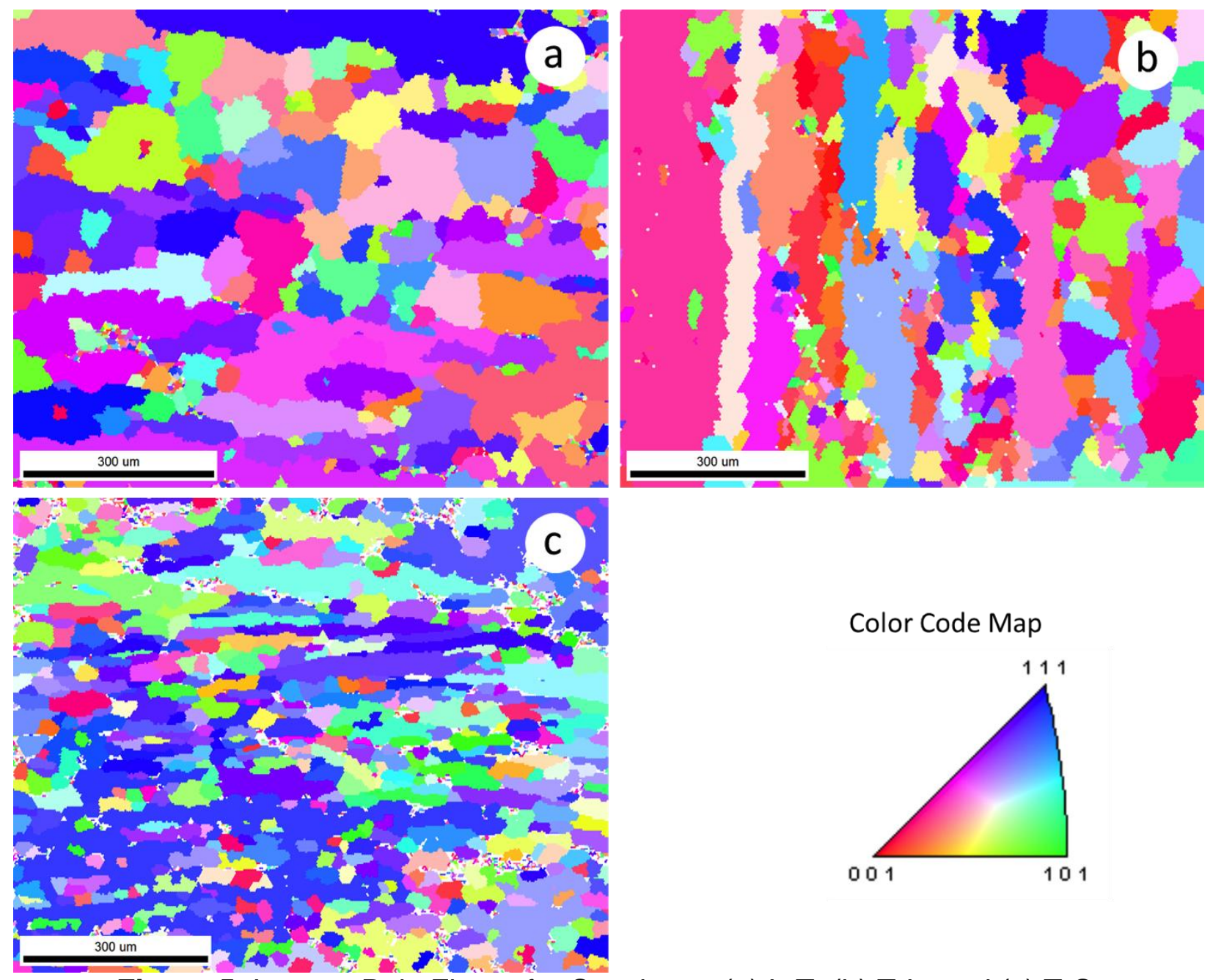

Figure 5. Inverse Pole Figure for Specimen: (a) L-T, (b) T-L, and (c) T-S.

Due to the substantial amount of second phase particles in AA 7050-T7451, caused by its overaged condition, some small areas in the inverse pole figure are just noise, since the EBSD does not account for precipitates. This noise was removed with standard filters available through the EBSD software package. The color map of the inverse pole figure does not represent the actual aluminum crystal orientation under these agglomerates, which are more likely to be a combination of $\mathrm{MgZn}_{2}, \mathrm{Al}_{7} \mathrm{Cu}_{2} \mathrm{Fe}$, $\mathrm{Al}_{2} \mathrm{CuMg}$, and $\mathrm{Mg}_{2} \mathrm{Si}$ [24]. The actual composition of each precipitate was not identified within the scope of the present work.

For all illustrations, the axial strain is shown in the horizontal direction. After achieving the imposed maximum strain, the specimen was unloaded, remaining with an overall plastic strain. The Correlated Solutions Software Vic-2D was used to perform the DIC in-plane measurements on the delimited area [23]. Figures 6-8 show the inplane strain maps for the three specimens (L-T, T-L, and T-S, respectively), with the actual axial, transverse and shear strains obtained with DIC analysis. 
It is important to note that the localized strain showed in each picture represents the residual strain upon unloading. This should not be confused with the residual elastic strains, normally obtained by diffraction techniques measuring lattice spacing. In the macroscale stress-strain relationship, the overall residual plastic strain is defined as the plastic strain; in the microscale, the localized residual plastic strain is the result of local accommodation of strain upon unloading, due to the anisotropy of the elastic tensor at crystal level and to the plastic anisotropy related to the specific orientation of the slip systems. A consequence of this crystal-level anisotropy is that the associated mechanical response such as crystallographic and morphologic texture, strength, strain hardening, deformation-induced surface roughening and damage are also orientation dependent [25]. The reference and deformed images were obtained at 10x and 20x magnification, for sensitivity analysis. The resolution obtained ranged from $0.3 \mu \mathrm{m} / \mathrm{pix}$ to $0.6 \mu \mathrm{m} / \mathrm{pix}$. To resolve the strain maps satisfactorily, with the $10 \mu \mathrm{m}$ base pattern, the subset size was $37 \mu \mathrm{m}$, with a step of $3 \mu \mathrm{m}$. The process for determining the subset size was by correlating two reference images, taken at different time at no load. The subset was consistently increased until a maximum of $0.1 \%$ of standard deviation was obtained for the entire correlated region. This procedure is necessary to avoid dipole of excessive strain $[29,30]$. It was noticed that there is no improvement in the resolved strain at 20x for this size of speckle, making the 10x magnification the optimum choice at that scale. The subset is about half size of the average grain. This allows a reasonable mapping of deformation at grain level. For the DIC performed at 10x magnification, more than 42,000 points were mapped to create the strain field.

The final total strain measured for the L-T specimen was $3.11 \%$, reaching 427 MPa. Fig. 6a shows the map of the residual plastic strain component along the loading direction with the superimposed grain boundaries determined by EBSD. The results from DIC show an average strain of $2.45 \%$. Despite this residual average strain, with standard deviation of $0.33 \%$, some regions could be resolved as having a much higher strain, showing localized strain concentration that can facilitate crack nucleation. The maximum plastic strain found was $3.20 \%$. At this resolution and after this amount of loading, no tendency could be obtained relating grain misalignment and strain concentration at the boundary. Fig. $6 \mathrm{~b}$ shows the residual transverse strain field, $\varepsilon_{y y}$, for the L-T specimen. The average DIC computed strain in this direction was found to be $0.74 \%$, with standard deviation of $0.21 \%$, in a range showed from $-2 \%$ to 0 . The in-plane shear strain field is shown in Fig. 6c. Ideally, the average for $\varepsilon_{x y}$ would be zero. The average $-0.15 \%$, with $0.25 \%$ standard deviation, found in the experiment can be explained by bias and uncertainties in the DIC measurements, by any small eccentricity in the load due to non-uniformed grip holding, and by the fact that the DIC analysis covers less than $5 \%$ of the total gauge section. For this case, $\varepsilon_{x y}$ strain values ranged from $-1.1 \%$ to $1.1 \%$. 




Figure 6. Specimen L-T residual (a) $\varepsilon_{x x}$ strain field. Average strain $=2.45 \%$; (b) $\varepsilon_{y y}$ strain field. Average strain $=-0.74 \%$; and (c) $\varepsilon_{x y}$ strain field. Average strain $=-0.15 \%$.

The T-L specimen was subjected to a $3.12 \%$ of total axial strain, reaching 489 MPa. The results from DIC showed $2.50 \%$ average axial strain for the analyzed area. Fig. $7 \mathrm{a}$ shows the map of residual plastic strains in the loading direction with the superimposed grain boundaries. For this case, the boundaries were represented for three different colors, according to the misalignment angle. Once again, we can visualize maximum localized residual plastic strain much higher than the average plastic strain, and lower strain regions with value less than half the computed mean strain. The strain range for this specimen is shown from $0.8 \%$ to $4.6 \%$, with average of $2.50 \%$ and 
$0.76 \%$ standard deviation. The maximum resolved strain and the scatter are higher than the values computed for the L-T specimen.

One interesting note is that there is a tendency for the axial strain to form isocurves extended perpendicular to the load direction. In the extreme left of Fig. 7a, there is a strip with high strain intensity. In this position, the EBSD resolved the topography as one long grain, what implies that any misalignment of the grains is lower than $5^{\circ}$. Right of this position, there is another strip with the lowest strain level. Even though this low strain band covers several identified grains over the $y$-direction, it can be seen that the predominance of misalignment angle between grains along the


the residual plastic strain field perpendicular to the loading direction, $\varepsilon_{y y}$, for the $T-L$ specimen. The average DIC strain in this direction was found to be $-0.90 \%$, with $0.23 \%$ standard deviation, in a range from $-2 \%$ to 0 . The in-plane shear strain field is shown in Fig. 7c. The average for $\varepsilon_{x y}$ in this case was found to be $0.05 \%$, with $0.30 \%$ standard deviation. 


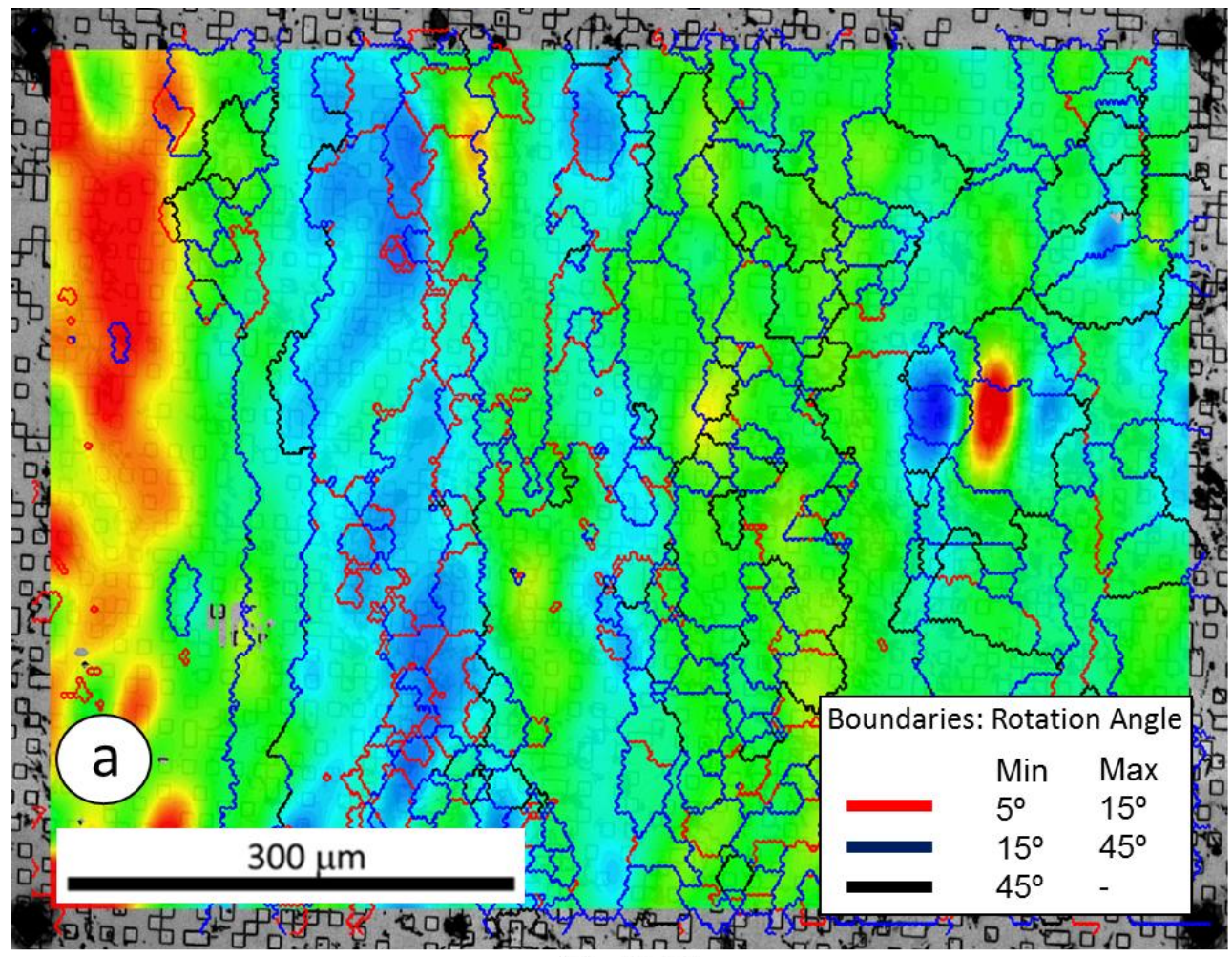

$\operatorname{exx}[\%]$
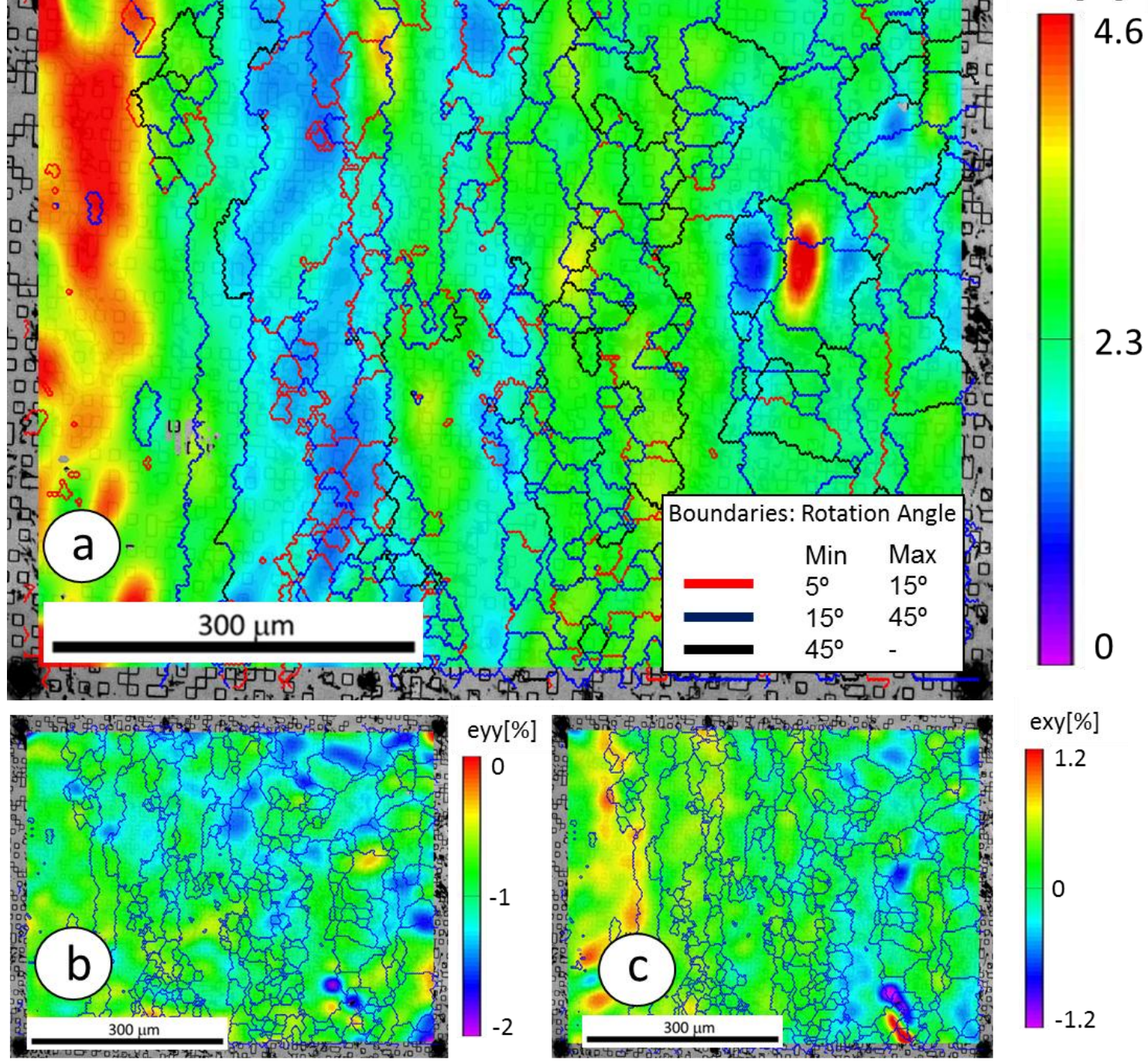

$\operatorname{exy}[\%]$

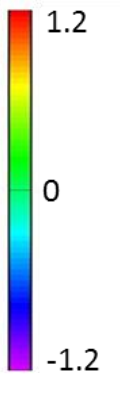

Figure 7. Specimen T-L residual (a) $\varepsilon_{x x}$ strain field. Average strain $=2.50 \%$; (b) $\varepsilon_{y y}$ strain field. Average strain $=-0.9 \%$; and (c) $\varepsilon_{x y}$ strain field. Average strain $=0.05 \%$.

The T-S specimen was subjected to a $3.10 \%$ of total axial strain, reaching 488 MPa. The results from DIC showed $2.26 \%$ average residual axial strain for the analyzed area. Fig. $8 \mathrm{a}$ shows the residual plastic strain component along the loading direction with the superimposed grain boundaries. The maximum localized residual axial strain shown is $3.8 \%$. The DIC average was $2.26 \%$ with $0.40 \%$ standard deviation. Fig. $8 b$ shows the residual transverse strain field, $\varepsilon_{y y}$, for the T-S specimen. The average DIC computed strain in this direction was found to be $-1.16 \%$, with $0.32 \%$ standard deviation, in a shown range in the saturated scale from $-3.0 \% 0$. The in-plane shear strain field is shown in Fig. 8c. The average for $\varepsilon_{x y}$ in this case was found to be $-0.10 \%$, with $0.24 \%$ standard deviation. These are the largest variations for the transverse strain among all specimens. For this specific case, the resolution of the resolved strain was 
compromised for the large base pattern size, which was $10 \mu \mathrm{m}$. More specifically for the T-S specimen, it is necessary to work with a finer speckle size. Once again is important to emphasize that the most significant result of the present work is a consistent and repeatable process to speckle a specimen for DIC. Even though the average strain in grain level is herein accurately determined, strain localization at sub grain level is in some sort compromised for the large size of the current pattern. However, at this resolution and speckle size, we still can resolve strain concentration caused by the precipitates on order of a few microns. The spots with localized high strain level shown in the figures are coincident with large precipitates present in the material.
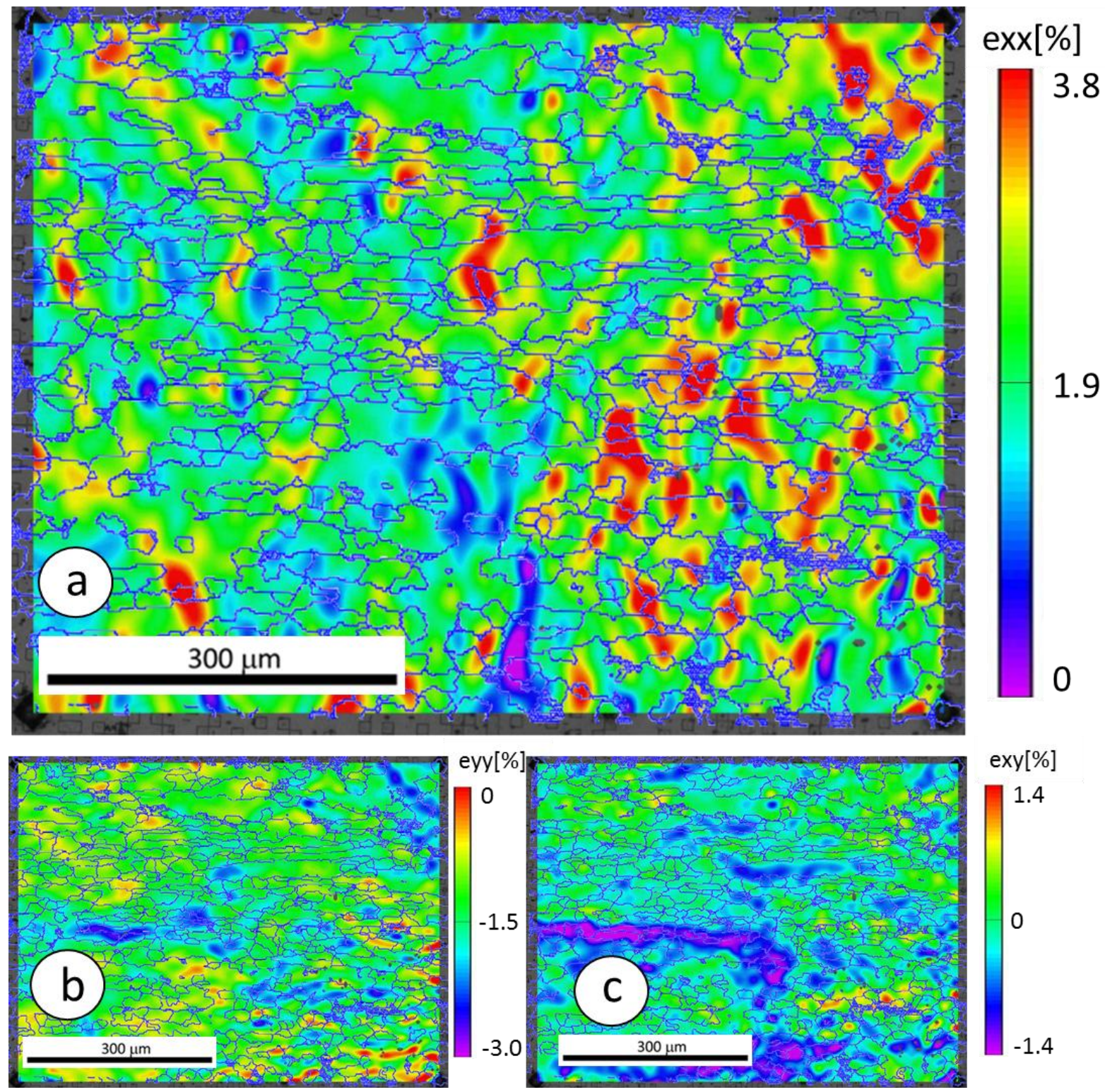

Figure 8. Specimen T-S residual (a) $\varepsilon_{x x}$ strain field. Average strain $=2.26 \%$; (b) $\varepsilon_{y y}$ strain field. Average strain $=-1.16 \%$; and (c) $\varepsilon_{x y}$ strain field. Average strain $=-0.10 \%$. 


\section{Simulations}

The conversion of the orientation data files from the EBSD to an appropriate input file for the simulations required several steps. The hexagonal gridded data inside each orientation file was converted into a square grid format. DREAM3D [22] has a filter that allows said conversion, though it must be noted that the conversion parameters, $x$ and $y$-spacing, have to be handled with care, as a large spacing will delete information and a small one will duplicate values enough times to fill the gaps. For all the simulations, the conversion parameters were smaller than the actual data spacing inside the file to prevent any data loss and ensure enough data points were added for each grain. Next, the data file was imported into an h5ebsd file, which allows for reference frame transformations to ensure that both the Euler and the spatial reference frames match. In this particular case, the Euler frame needed to be rotated $90^{\circ}$ about the $<001>$ so that it could match the spatial frame. The data was also screened and corrected for any bad data point encountered. A point is considered to be bad if both the confidence index is below 0.2 and the misorientation angle relative to its neighbors surpasses $5^{\circ}$ (which is commonly encountered for precipitates that appear on the specimen's surface). With the final corrected and rotated data, the simulation file was generated, containing the orientation angles, the spatial locations, the grain ID, and the phase to which it belongs. Note that we do not explicitly account for the precipitates, since this work focuses on the role of the grain morphology on the strain localization. We note that the stress-strain curves in Fig. 4 implicitly capture the mechanical response of the precipitates, which is used to fit the Voce hardening law (Eq. 16). Thus, we average the hardening response of the precipitates over the slip systems, but do not explicitly include the precipitates within the microstructure of the crystal plasticity simulations. Future work will investigate precipitate morphology and orientation but is beyond the scope of the current paper.

Anisotropic elastic constants for AA 7050 were obtained from Pereira et al. [26]. It is noted that no appreciable difference was observed in the strain maps from using anisotropic versus isotropic elasticity. For the case of isotropy, a Young's modulus of $72,448 \mathrm{MPa}$ was directly obtained from the experimental stress-strain curves and a Poisson ratio of 0.33 was approximated. Fig. 9 shows the macroscopic stress-strain relationship for the three specimen orientations for obtaining the generalized hardening law parameters. The final Voce hardening parameters from microscopic fitting are shown in Table 1.

The FFT-based formulation allows the imposition of a macroscopic strain rate, a macroscopic stress, or a combination of both as long as they are complementary. For this model, only the macroscopic strain rate, $\dot{E}$, along the $x$-direction was imposed, while the other strain components were adjusted to fulfill stress-free conditions for the corresponding stress components.

The microstructure profile was reconstructed from the three EBSD scans (L-T, TL, T-S) and was modeled as a through-thickness columnar grain structure by leaving a 
1 voxel thickness, so that the periodicity of FFT could evaluate the grains on the EBSD as extruded to infinity. Thus, three different unit cells are created as input to the simulations, assuming columnar grains in the third direction. We note this assumption is most valid in the case of the T-S sample, as the pancake shaped grains are elongated in the $L$ direction due to the rolling process. But in general, given that each voxel is $\sim 3$ microns deep and that the actual thickness of the specimen is larger by several orders of magnitude, an infinitely columnar structure is a decent assumption during modeling of even the L-T and T-L samples.

The minimum representative size for each specimen was found to be (i) $T-L=$ $232 \times 190 \times 1$ voxels $=1000 \times 700 \times 3.7$ microns; (ii) $\mathrm{L}-\mathrm{T}=361 \times 290 \times 1$ voxels $=938 \times 754 \times 2.6$ microns, and (iii) T-S $=372 \times 297 \times 1$ voxels $=967 \times 772 \times 2.6$ microns. Given the fact that the simulation requires a periodic microstructure, a gas phase was added on the free boundaries and extra material was added on the constrained ones to achieve periodicity. Neither of these additional boundary constraints will be considered on the strain results shown in this section. With the gas phase and extra material added, the minimum size needed for a representative T-L simulation was found to be $256 \times 256 \times 1$ voxels, while for L-T and T-S the size had to be at least $512 \times 512 \times 1$ voxels. To ensure quality on the results, a final size of $1024 \times 1024 \times 1$ was used on all models. The increase in size had no impact on the macroscopic behavior. However, it added resolution to the results of each sample's microstructural behavior.

Table 1. Voce's Hardening Parameters for AA 7050-T7451 (MPa).

\begin{tabular}{|c|c|c|c|}
\hline$\tau_{0}$ & $\boldsymbol{\theta}_{0}$ & $\tau_{1}$ & $\boldsymbol{\theta}_{1}$ \\
\hline 135.8337 & 3061.5587 & 8.4697 & 107.586 \\
\hline
\end{tabular}




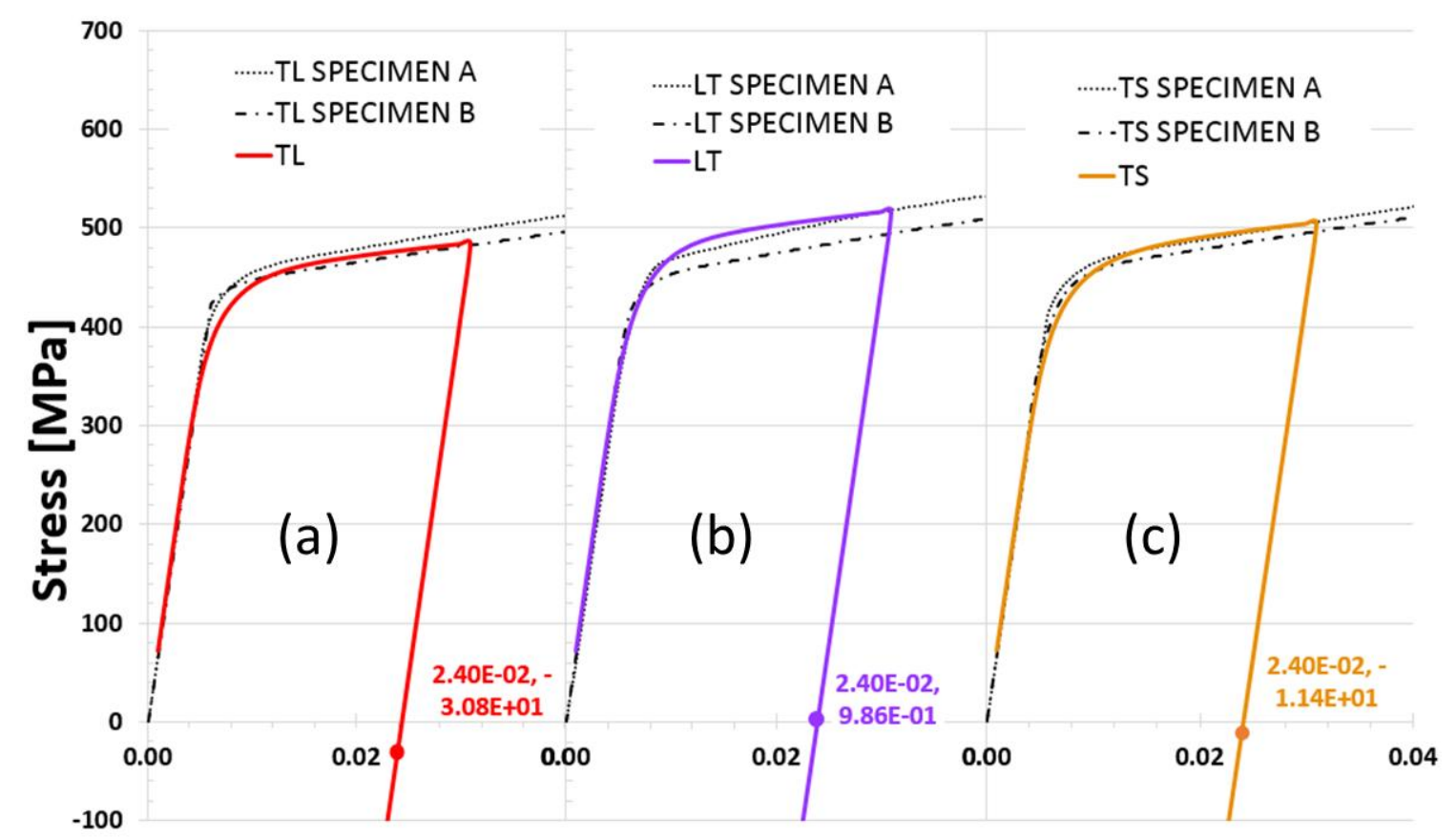

Strain

Figure 9. Stress-strain fittings for macroscopic level model calibration for (a) T-L, (b) L-T, and (c) T-S Specimens.

\section{Discussion}

Figure 10 shows the simulated strain field maps side-to-side with the experimental measurements. As it can be observed, the simulations do not capture the exact heterogeneous strain distributions at the microstructural features as represented in the DIC-EBSD experiments, especially for the L-T and T-S specimens. However, the simulations correctly predict the statistical strain distributions for each crystallographic texture. Fig. 11 shows the histograms and cumulative distribution functions obtained for each specimen, comparing simulation with experimental data. It can be seen in each case that at a nominal residual plastic strain of approximately $\sim 2.4 \%$, multiple locations exhibit strains greater than $5 \%$ (roughly double the applied macroscopic strain). This is quite an important ramification for design and structural analysis: even macroscopic loadings that are firmly within the elastic region of the material's behavior may create local plastic strains, thus resulting in hot spots that are prone to failure, especially for fatigue. The crystal plasticity simulation correctly predicted the complete strain distributions, which are important to predict failure. Within an engineering context, the accurate prediction of statistical distributions of local strains is important for materials and structural analysis of an ensemble of components.

In general, the model results show that the strain concentration levels are in statistical agreement with the experiments. However, the strain distribution at the individual microstructural features from the simulations did not match the DIC results. 
The possible sources for these differences are explained as follows: (i) the different boundary conditions between the experiment and model, (ii) the role of the subsurface microstructure on the surface strain response, (iii) incomplete physics of the constitutive equations used in the materials models, and (iv) the resolution from $10 \mu \mathrm{m}$ pattern DIC analysis.

Regarding the boundary conditions, the simulation corresponds to a uniform average strain rate throughout the entire material, whereas experimentally this strain rate is only applied to the grips of the specimens. In the gauge section of the specimen, where the strain maps are characterized, the neighboring grains govern the exact boundary conditions. In other words, unlike the simulation the deformation is not truly uniform on the actual specimen. With respect to the subsurface microstructure, the computational model maps the surface grains and applies periodic boundary conditions (this is commonly called a 2.5 dimensional model). Thus, the model assumes grains to be columnar, which means that the underlying layers are formed by exactly the same structure shown on the surface. As demonstrated by Turner et al. [27], the 3-D subsurface features will have a considerable effect on the surface strain. Now, since the actual underlying grain structure is unknown, a discrepancy between the observed strain fields was expected. Nonetheless, further study shall be required to gauge the extent in which the columnar assumption affects the results. The work of Tasan et al. [10] and Lim et al. [11] demonstrates oligocrystals can be used to mitigate concerns surrounding subsurface effects and result in better agreement between simulated and measure surface strain fields.

The constitutive equations used within the crystal plasticity model may be incomplete. For instance, the exact form of the flow rule ignores the role of normal stress on the slip system or hydrostatic stresses. It should be noted that the crystal plasticity formulation used in this study does not include a characteristic length-scale, thus we cannot account for grain sizes within the microstructure. Given the pancake shaped grains in this rolled microstructure, the aspect ratio of the grains may have size effects on the resulting strain maps. Strain-gradient approaches would be necessary to account for these size effects. The EVP-FFT formulation does take into account grain morphology. Therefore, as earlier noted, we would expect the T-S model to be most accurate, since the pancake shaped grains are elongated normal to the T-S direction, thus the assumption of columnar grains is easily justified for this orientation. Additionally, the generalized Voce law assumes that all slip systems harden an equal amount, which ignores dislocation pile-ups on individual slip systems. It is also possible that, by using this isotropic Voce hardening law, previous directional hardening caused by the rolling of the material is not being taken into account when modeling strain on the TL-LT-TS directions. Thus, the residual strain distributions within the grains, prior to loading, should be orientation dependent due to the rolling process. Also, the Voce law reduces the amount of variables involved in the calculation, which in turn lowers the level of parametric uncertainty of the model and therefore benefits the computational time required to fit and run the simulations. For multiple hardening laws commonly employed, the resulting slip system activity can be quite drastic while satisfying the 
same macroscopic response [28]. Since the form of the constitutive laws used in crystal plasticity simulations are not widely agreed upon, it should be expected that these equations would result in model form error (also known as epistemic), which will propagate to errors in the model predictions.



(a)
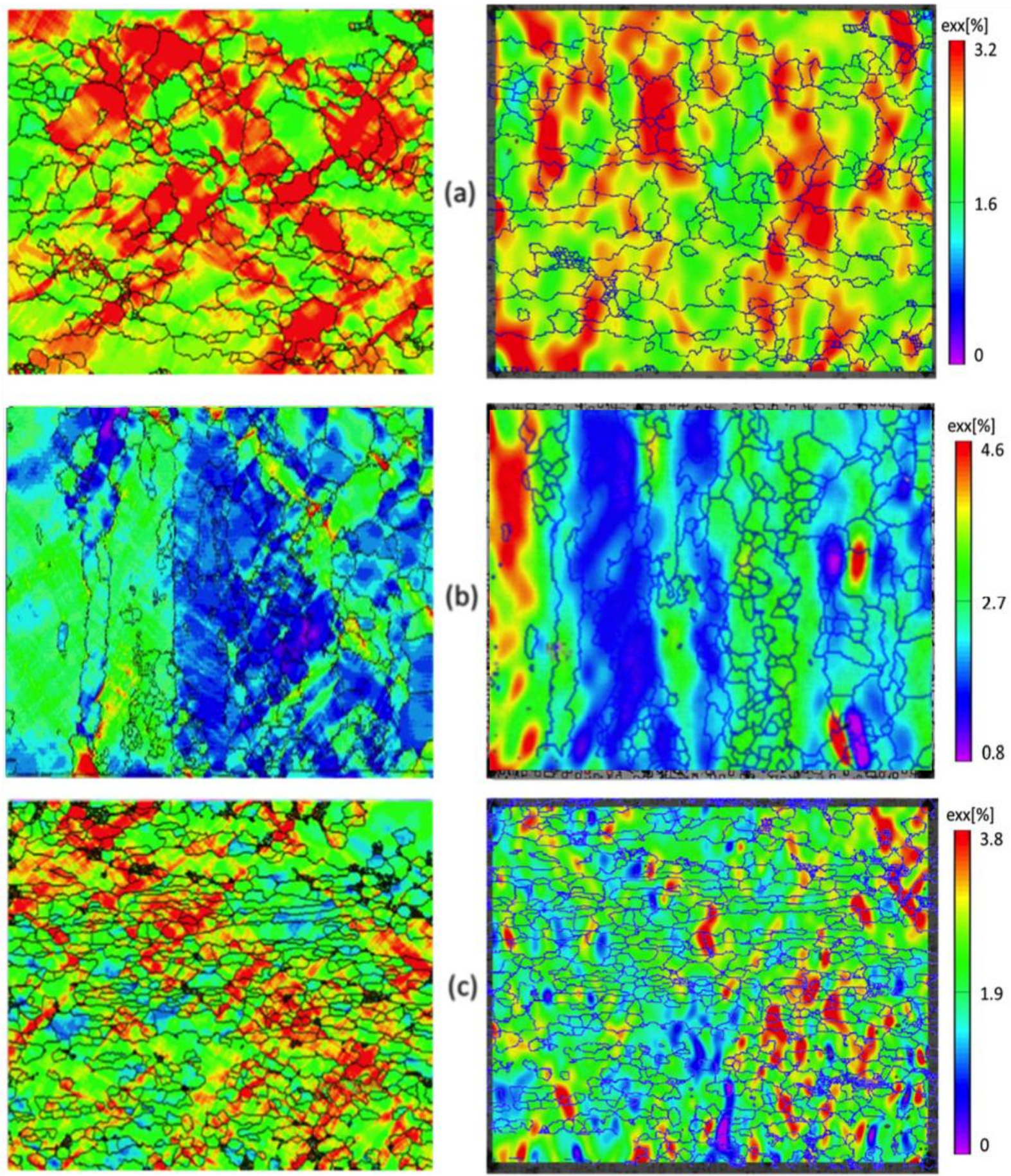

Figure 10. Simulation (left) vs. experimental (right) results for (a) L-T, (b) T-L, and (c) TS specimens. 

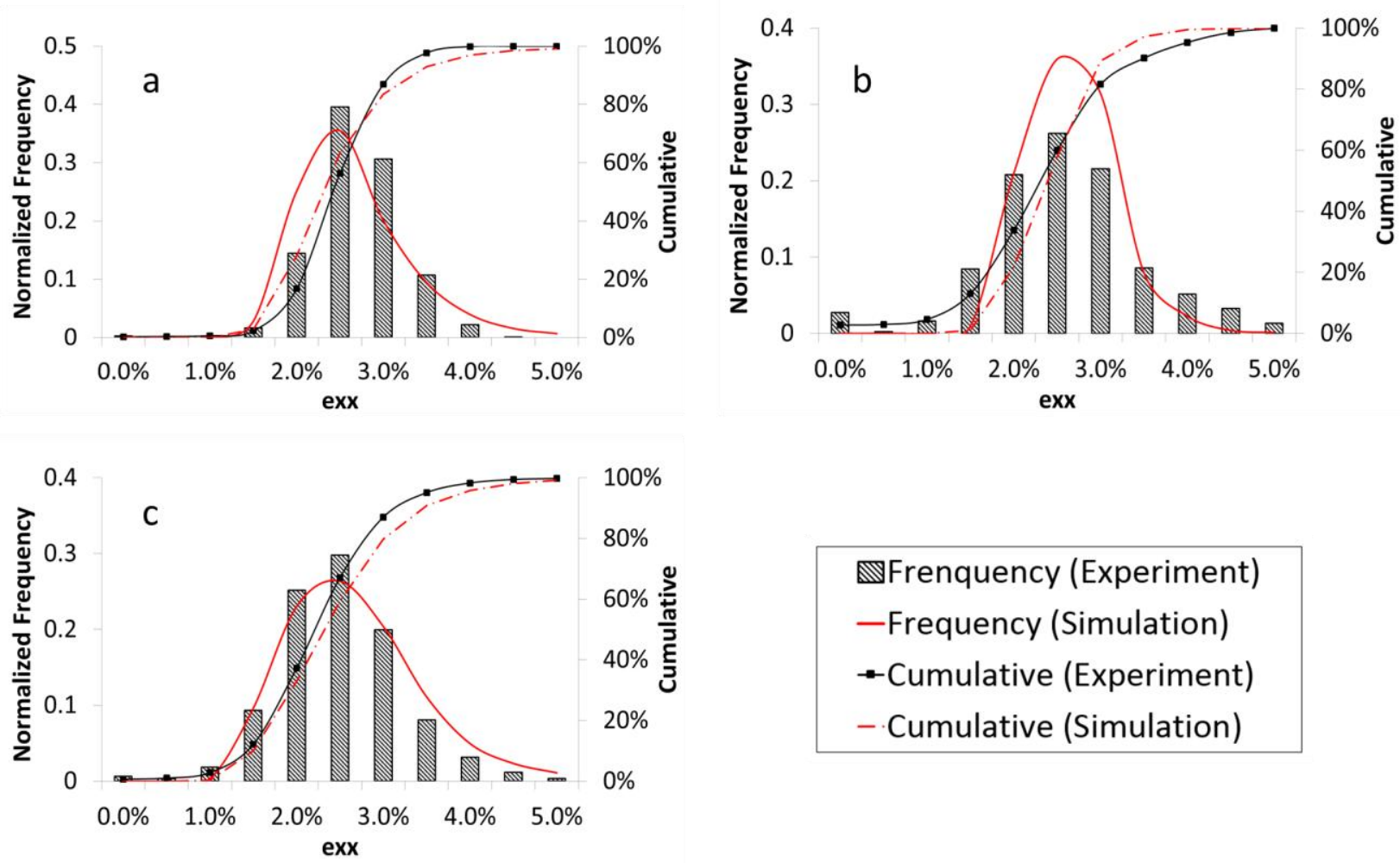

$\mathbb{Q}$ Frenquency (Experiment)

-Frequency (Simulation)

-Cumulative (Experiment)

- Cumulative (Simulation)

Figure 11. Histograms of axial plastic strain distribution for the (a) L-T, (b) T-L, and (c) T-S Specimen.

\section{Conclusion}

The novel micro-stamping used in the present work has been shown to be very effective for digital image correlation. The $10 \mu \mathrm{m}$ base pattern element was sufficient to provide grain level resolution for the AA 7050 T-7451, at 10x optical resolution. For subgrain resolution, it is recommended that a finer pattern be used and imaged at higher magnification. The process is totally controlled and repeatable, what justify efforts in development and production of finer stamps, on the order of micron or submicron. As can be seen from the strain field maps, the strain varies according to grain orientation and it is clearly affected by the neighbor grains. There is a tendency for the axial strain to form isocurves extended perpendicular to the load direction. This is more noticeable for the T-L specimen where the grains are elongated in that direction, favoring the 
condition. The experiments have shown that the maximum residual plastic strain at grain level was about twice the average residual plastic strain on the specimen.

An EVP-FFT simulation was used to model uniaxial loading with respect to three different orientations of rolled AA 7050-T7451. The statistical nature of the strain fields were reasonably well predicted by the EVP-FFT simulation, as the maximum microstructural strains were roughly double the macroscopic residual plastic strains. This result has a profound influence on the material design that may experience failure (especially from cyclic or time dependent loadings) at stresses well below the macroscopic yield point of the material. It is noted that crystal plasticity could not accurately predict the heterogeneous strain fields at each microstructural features. A better match between model and real media has to be pursued for improving the prediction of spatial strain maps across the microstructure, as several reasons for discrepancies between the model and the experiment were discussed. In many engineering applications, it is more important to capture the statistical nature of heterogeneous strain, compared with matching the strain distribution at each microstructure feature, as the strain statistics can be used to predict the life of an ensemble of components.

\section{Acknowledgements}

The authors gratefully acknowledge funding from the Office of Naval Research, N00014-14-1-0544. RAL acknowledges support from the Joint DoD/DOE Munitions Technology Programs. Also, the authors graciously acknowledge technical support and advice from Mr. Andrea Rovinelli (EVP-FFT) and Major Todd Book (EBSD). Drs. Jacob Hochhalter and Andrew Cannon are thanked for their help with the micro stamping design, development, and procedure.

\section{Appendix}

Table 2. Mechanical Properties - AA 7050-T7451, $3 \mathrm{~mm}$ by $1.6 \mathrm{~mm}$ cross section.

\begin{tabular}{|c|c|c|c|c|c|c|c|}
\hline \multirow{2}{*}{ Property \Specimen } & & \multicolumn{2}{|c|}{ L-T } & \multicolumn{2}{|c|}{ T-L } & \multicolumn{2}{|c|}{ T-S } \\
\hline & Unit & A & B & A & B & A & B \\
\hline Hardness, Vickers & HV5 & 176 & 178 & 178 & 183 & 175 & 175 \\
\hline Elongation at Yield & $\%$ & $0.84 \%$ & $0.82 \%$ & $0.81 \%$ & $0.83 \%$ & $0.82 \%$ & $0.81 \%$ \\
\hline Elongation at Break & $\%$ & $11.0 \%$ & $9.0 \%$ & $11.0 \%$ & $15.0 \%$ & $15.0 \%$ & $14.0 \%$ \\
\hline Reduction in Area (neck) & $\%$ & $18.7 \%$ & $13.2 \%$ & $14.7 \%$ & $19.0 \%$ & $22.6 \%$ & $26.3 \%$ \\
\hline Ultimate Tensile Strenght & $\mathrm{MPa}$ & 516.9 & 506.2 & 507.4 & 490.7 & 517.6 & 508.8 \\
\hline Tensile Yield Strenght $(0.2 \%)$ & $\mathrm{MPa}$ & 455.6 & 440.0 & 438.4 & 437.3 & 448.5 & 438.8 \\
\hline Modulus of Elasticity & Gpa & 71.0 & 71.4 & 71.9 & 69.7 & 71.9 & 72.1 \\
\hline Strain at maximum load & $\%$ & $6.4 \%$ & $8.7 \%$ & $7.6 \%$ & $7.3 \%$ & $7.8 \%$ & $8.0 \%$ \\
\hline
\end{tabular}




\section{References}

[1] Tschopp, M.A., Bartha, B.B., Porter, W.J., Murray, P.T., Fairchild, S.B., 2009. Microstructure-Dependent local strain behavior in polycrystals through In-Situ scanning electron microscope tensile experiments. Metall. Mater. Trans. A Phys. Metall. Mater. Sci. 40, 2363-2368. doi:10.1007/s11661-009-9938-6

[2] Clair, A., Foucault, M., Calonne, O., Lacroute, Y., Markey, L., Salazar, M., Vignal, V., Finot, E., 2011. Strain mapping near a triple junction in strained Ni-based alloy using EBSD and biaxial nanogauges. Acta Mater. 59, 3116-3123. doi:10.1016/j.actamat.2011.01.051

[3] Abuzaid, W.Z., Sangid, M.D., Carroll, J.D., Sehitoglu, H., Lambros, J., 2012. Slip transfer and plastic strain accumulation across grain boundaries in Hastelloy $X$. J. Mech. Phys. Solids 60, 1201-1220. doi:10.1016/j.jmps.2012.02.001

[4] J. L. Carter, M. W. Kuper, M. D. Uchic, M. J. Mills, 2014. Characterization of localized deformation near grain boundaries of superalloy Rene-104 at elevated temperatures. Mat. Sci. \& Eng. A 605, 127-136. doi:10.1016/j.msea.2014.03.048

[5] Kammers, A.D., Daly, S., 2013. Self-Assembled Nanoparticle Surface Patterning for Improved Digital Image Correlation in a Scanning Electron Microscope. Exp. Mech. 53, 1333-1341. doi:10.1007/s11340-013-9734-5

[6] Di Gioacchino, F., Quinta da Fonseca, J., 2012. Plastic Strain Mapping with Submicron Resolution Using Digital Image Correlation. Exp Mech 53, 743-754. doi:10.1007/s11340-012-9685-2

[7] Esquivel J., Sangid M.D., 2015. Digital image correlation of heterogeneous deformation in polycrystalline material with electron backscatter diffraction, 2015 Microscopy and Microanalysis Conference, August 5, 2015, Portland, Oregon. 0546-000591.

[8] Zhao, Z., Ramesh, M., Raabe, D., Cuitiño, A., Radovitzky, R., 2008. Investigation of three-dimensional aspects of grain-scale plastic surface deformation of an aluminum oligocrystal. Int. J. Plast. 24, 2278-2297. doi:10.1016/j.jplas.2008.01.002

[9] Turner, T.J., Shade, P. a, Schuren, J.C., Groeber, M. a, 2013. The influence of microstructure on surface strain distributions in a nickel micro-tension specimen. Model. Simul. Mater. Sci. Eng. 21, 015002. doi:10.1088/0965-0393/21/1/015002

[10] Tasan, C.C., Hoefnagels, J.P.M., Diehl, M., Yan, D., Roters, F., Raabe, D., 2014. Strain localization and damage in dual phase steels investigated by coupled insitu deformation experiments and crystal plasticity simulations. Int. J. Plast. In press. doi:10.1016/j.ijplas.2014.06.004

[11] Lim, H., Carroll, J., Battaile, C., Boyce, B., Weinberger, C., 2015. Quantitative comparison between experimental measurements and CP-FEM predictions of plastic deformation in a tantalum oligocrystal. International Journal of Mechanical Sciences 92, 98-108. doi:10.1016/j.ijmecsci.2014.12.010

[12] AMS 4050J, 2014. Aluminum Alloy, Plate, 6.2Zn-2.3Cu - 2.2Mg - 0.12Zr (7050T7451), Solution Heat Treated, Stress Relieved, and Overaged. SAE International. 
[13] ASTM E8 / E8M-13a, 2013. Standard Test Methods for Tension Testing of Metallic Materials, ASTM Int. 1-28. doi:10.1520/E0008_E0008M-11

[14] Efstathiou, C., Sehitoglu, H., Lambros, J., 2010. Multiscale strain measurements of plastically deforming polycrystalline titanium: Role of deformation heterogeneities. Int. J. Plast. 26, 93-106. doi:10.1016/j.ijplas.2009.04.006

[15] Cannon, A.H., Macguire, M.C., Hochhalter, J.D., 2015. US Patent Application 62116742.

[16] Cannon, A.H., King, W.P., 2009. Casting metal microstructures from a flexible and reusable mold. J. Micromechanics Microengineering. doi:10.1088/09601317/19/9/095016

[17] Cannon, A.H., Hochhalter, J.D., Mello, A.W., Bomarito, G.F., Sangid, M.D., 2015. MicroStamping for Improved Speckle Patterns to Enable Digital Image Correlation. 2015 Microscopy and Microanalysis Conference, August 4, 2015, Portland, Oregon. ID: 0546-000769.

[18] Lebensohn, R.A., Kanjarla, A.K., Eisenlohr, P., 2012. An elasto-viscoplastic formulation based on fast Fourier transforms for the prediction of micromechanical fields in polycrystalline materials. Int. J. Plast. 32-33, 59-69. doi:10.1016/j.ijplas.2011.12.005

[19] Moulinec, H., Suquet, P., 1998. A numerical method for computing the overall response of nonlinear composites with complex microstructure. Comput. Methods Appl. Mech. Eng. doi:10.1016/S0045-7825(97)00218-1

[20] Liu, B., Raabe, D., Roters, F., Eisenlohr, P., Lebensohn, R.A., 2010. Comparison of finite element and fast Fourier transform crystal plasticity solvers for texture prediction. Model. Simul. Mater. Sci. Eng. doi:10.1088/0965-0393/18/8/085005

[21] Tome, C., Canova, G.R., Kocks, U.F., Christodoulou, N., Jonas, J.J., 1984. The relation between macroscopic and microscopic strain hardening in F.C.C. polycrystals. Acta Metall. doi:10.1016/0001-6160(84)90222-0

[22] Groeber, M., Rollett, T., Jackson, M., Rowenhorst, D., Rohrer, G., 2015. Dream3D. Bluequartz Software. http://dream3d.bluequartz.net

[23] Sutton, M., Wolters, W., Peters, W., Ranson, W., McNeill, S., 1983. Determination of displacements using an improved digital correlation method. Image Vis. Comput. doi:10.1016/0262-8856(83)90064-1

[24] Crawford, B.R., Sharp, P.K., 2012. Equivalent Crack Size Modelling of Corrosion Pitting in an AA7050-T7451 Aluminum Alloy and its Implications for Aircraft Structural Integrity. Air Vehicles Division. Defence Science and Technology Organisation. DSTO-TR-2745. Australia.

[25] Roters, F., Eisenlohr, P., Hantcherli, L., Tjahjanto, D.D., Bieler, T.R., Raabe, D., 2010. Overview of constitutive laws, kinematics, homogenization and multiscale methods in crystal plasticity finite-element modeling: Theory, experiments, applications. Acta Mater. 58, 1152-1211. doi:10.1016/j.actamat.2009.10.058

[26] Pereira, P., Santos, A., 2012. Influence of Anisotropy Generated by Rolling on the Stress Measurement by Ultrasound in 7050 T7451 Aluminum. Exp Mech 53, 415-425. doi:10.1007/s11340-012-9647-8 
[27] Turner, T.J., Shade, P. a, Schuren, J.C., Groeber, M. a, 2013. The influence of microstructure on surface strain distributions in a nickel micro-tension specimen. Model. Simul. Mater. Sci. Eng. 21, 015002. doi:10.1088/0965-0393/21/1/015002

[28] Sangid, M.D., Yeratapally, S.R., Rovinelli, A, 2014. Validation of MicrostructureBased Materials Modeling. American Institute of Aeronautics and Astronautics Journal. 55th AIAA/ASMe/ASCE/AHS/SC Structures, Structural Dynamics, and Materials Conference. January 14, 2014, National Harbor, Maryland. DOI: 10.2514/6.2014-0462.

[29] Efstathiou, C. at al. 2010. Multiscale strain measurements of plastically deforming polycrystalline titanium: Role of deformation heterogeneities. International Journal of Plasticity. doi:10.1016/j.ijplas.2009.04.006.

[30] Padilla, H.A. et al. 1012. Relating inhomogeneous deformation to local texture in zirconium through grain-scale digital image correlation strain mapping experiments. doi:10.1016/j.ijsolstr.2011.09.001 